\title{
A Memetic Approach to Vehicle Routing Problem with Dynamic Requests
}

\author{
Jacek Mańdziuk ${ }^{\mathrm{a}, \mathrm{b}, *}$, Adam Żychowski ${ }^{\mathrm{a}}$ \\ ${ }^{a}$ Faculty of Mathematics and Information Science, Warsaw University of Technology, \\ Koszykowa 75, 00-662 Warsaw, Poland \\ ${ }^{b}$ School of Computer Engineering, Nanyang Technological University, \\ Block N4, Nanyang Avenue, Singapore 639798
}

\begin{abstract}
The paper presents an effective algorithm for solving Vehicle Routing Problem with Dynamic Requests based on memetic algorithms. The proposed method is applied to a widely-used set of 21 benchmark problems yielding 14 new bestknow results when using the same numbers of fitness function evaluations as the comparative methods. Apart from encouraging numerical outcomes, the main contribution of the paper is investigation into the importance of the so-called starting delay parameter, whose appropriate selection has a crucial impact on the quality of results. Another key factor in accomplishing high quality results is attributed to the proposed effective mechanism of knowledge transfer between partial solutions developed in consecutive time slices. While particular problem encoding and memetic local optimization scheme were already presented in the literature, the novelty of this work lies in their innovative combination into one synergetic system as well as their application to a different problem than in the original works.
\end{abstract}

Keywords: vehicle routing, memetic algorithm, optimization under uncertainty, scheduling, dynamic optimization

\footnotetext{
*Corresponding author

Email address: mandziuk@mini.pw.edu.pl (Jacek Mańdziuk)
} 


\section{Introduction}

Vehicle Routing Problem (VRP), first introduced by Dantzig and Ramser in [1, is an NP-hard combinatorial optimization problem with many practical applications. In its classical formulation a set of customers must be served by a ning and ending at a specified depot. The optimization goal is to minimize the total routes' length/cost of all vehicles. Due to the VRP's combinatorial complexity the exact solution methods proved inefficient, except for simple problems. Therefore most of the research effort was devoted to application of metaheuris(TS) 3, Genetic Algorithms (GA) [4, Simulated Annealing (SA) [5] or Upper Confidence Bounds Applied to Trees (UCT) [6], with promising results.

In majority of practical applications, however, another dimension related to stochastic nature of the real-life problems is added to the problem's formu15 lation. In particular, the information available at the beginning may change during the solution execution, for instance due to the arrival of new customers' orders [7, 8] or because of the changes in their requested demands [9] or because of unplanned events, e.g. traffic jams/road congestion [10, 11] or, more generally, due to stochastic travel times [12. This intrinsic stochasticity of the Dynamic Vehicle Routing Problems (DVRP), solving which requires adequate on-line routes' adjustment in response to the arrival of new problem-related information.

Furthermore, in the literature there are many variants of the VRP which are formulated so as to serve specific practical needs, e.g. its multi-trip [13] or multicompartment [14] versions, variants with specific time-windows for delivery [15], formulations which combine delivery with picking up goods from the clients [16, and many others (see the recent special issue [17 for an overview of the current developments and challenges in this domain and [18 for the VRP taxonomy).

This paper considers a version of DVRP in which some of the customers' 
locations (and their associated requests) are unknown at the start of the solving method and arrive gradually as time passes, i.e. during the algorithm execution. In the literature, this version of DVRP is known as Vehicle Routing Problem with Dynamic Requests (VRPDR). The intrinsic partial information uncertainty of VRPDR is usually handled in the way proposed by Kilby et al. [8], which consists in executing the route optimization procedure only at the end of predefined fixed time intervals - called time slices.

In general, the algorithms applied to solving VRPDR are similar to those used for VRP. In particular, Computational Intelligence methods, e.g. ACO [19],

40 TS [20], GA [20] or PSO [21, 22], have been applied to the problem with some success. Specifically, the PSO based approaches [23, 24, 22, 25], seem to be very well suited to the type of dynamic changes introduced to requests' distribution observed in VRPDR. This issue is further discussed in Section [5] devoted to presentation of experimental results.

45 The approach proposed in this paper is based on Memetic Computing (MC) 26 27], which is currently one of the fastest growing subfields of Evolutionary Computing research. In short, MC enhances population-based Evolutionary Algorithms (EA) by means of adding a distinctive local optimization phase. The underpinning idea of $\mathrm{MC}$ is to use domain knowledge or local optimization techniques to improve potential solutions (represented by individuals in a population) between consecutive EA generations. A synergetic combination of simple local improvement schemes called memes with evolutionary operators leads to complex and powerful solving paradigm, applicable to a wide range of problems 28].

MC has been applied to several variants of transportation problems, in particular the static VRP version [29, 30] or the Vehicle Routing Problem with Stochastic Demands (VRPSD) 9]. However, to the best of our knowledge, this paper presents the first attempt to solving VRPDR with MC. On the other hand our approach, to some extent, follows the above-cited work of Chen et al., ${ }_{60}$ even though the problems considered in these two papers are quite different. In VRPSD, unlike in VRPDR, all customers' locations are known in advance (at 
the start of the method) but customers' demands are stochastic, i.e. the size of demand is known only after the vehicle's arrival. Hence, the main focus of the solution method is to ensure that a planned route would not exceed vehicle's capacity.

Another paper that inspired our work is [20] where simple but powerful chromosome representation and genetic operators were proposed. While a unique combination of the MC scheme adopted from [9] with solution representation proposed in 20. proved to be superior over each of the two components alone, the respective results were, anyway, not better than those accomplished with the 2MPSO (Two-Phase Multiswarm PSO) method [22, 25]. Only after the proposed method was enhanced by a suitable starting delay mechanism and the effective way of knowledge transfer between consecutive time slices, the final results yielded by the system excelled those of $2 \mathrm{MPSO}$ in terms of the average 75 performance and the best minima found.

The main contribution of this paper, except for finding new best-literature results for popular benchmarks is investigation into the saliency of the starting delay parameter. Another key issues are introduction of a new effective way of knowledge transfer between consecutive time slices by means of a specific population generation scheme, as well as, introduction of a new mutation operator in the memetic optimization procedure. The novelty of this work also lies in the innovative synergetic combination of (already known in the literature) problem encoding and memetic optimization and their application to a new problem from VRP domain.

The remainder of this paper is arranged as follows. Section 2 presents the VRPDR definition and discusses its practical relevance. In Section 3 general overview of the system's construction, its main principles, as well as basic components (each in a dedicated subsection) are presented. Section 4 provides benchmarks description, discussion on experimental methodology and parameters' selection. In Section 5 experimental results are presented and discussed in the context of the best literature solutions, in particular those accomplished with the 2MPSO algorithm [21, 22, 25]. Performance analysis of the proposed 
system and discussion on its suitability for particular types of benchmark sets are also placed in this section. The next section elaborates on the pertinence of the local memetic optimization component and the saliency of the method's steering parameters. The last section is devoted to conclusions and directions for future research.

\section{Definition of VRPDR}

VRPDR is a generalization of the Traveling Salesman Problem. In this problem a fleet of $m$ homogenous vehicles, each with identical capacity $c$, and the set of $n$ customers $\left\{v_{1}, v_{2}, \ldots, v_{n}\right\}$ are considered. VRPDR can be modeled using an undirected graph $G=(V, E)$, where $V=\left\{v_{0}, v_{1}, \ldots, v_{n}\right\}$ is the vertex set and $E=\left\{\left(v_{i}, v_{j}\right): v_{i}, v_{j} \in V, i<j\right\}$ is the edge set. Each vertex $v_{i}, i=1, \ldots, n$ represents the respective $\left(i\right.$-th) customer and $v_{0}$ denotes a depot. Each edge $e_{i j}=\left(v_{i}, v_{j}\right), i, j=0, \ldots, n$ has an associated weight which represents the cost or, alternatively, a distance between $v_{i}$ and $v_{j}$ being either two customers or a customer and the depot. Furthermore, for each customer $v_{i}$ the demand $d_{i}$, the unload time $u t_{i}$ (which is the time required to unload cargo at customer's $v_{i}$ ) and $t_{v_{i}}$ - the time of arrival of the order from customer $v_{i}$, are defined. Depot $v_{0}$ has the opening time $t_{o}$ and closing time $t_{c},\left(0 \leq t_{o}<t_{c}\right)$ specified. The speed of each vehicle is defined as one distance unit per one time unit.

The goal of VRPDR is to minimize the total routes' length of all vehicles according to the following constraints:

- each vehicle has to start from a depot after time $t_{o}$ and end its route in a depot before time $t_{c}$,

- every customer has to be served exactly once and by one vehicle,

- time of a vehicle's arrival to customer $v_{i}$ has to be greater than $t_{v_{i}}$ for all $i$

- the sum of customers' demands assigned to each vehicle must not exceed vehicle's capacity $c$. 
VRPDR combines two NP-Complete problems: Bin Packing Problem (to assign requests to vehicles) and Traveling Salesman Problem (to minimize the tour length of each vehicle). The problem is widely applicable to real-life tasks, such as taxi services, courier companies or other pickup and delivery businesses. The Global Positioning System and the widespread use of mobile phones create opportunity for companies to track and manage their fleet in real time, thus making the VRPDR a highly relevant problem of practical importance.

\section{Components of the system}

Our system designed to solving the VRPDR is composed of two main components. The first module is responsible for receiving new orders, dividing working day into some pre-defined number of time slices and creating static instances of the VRP for each of them. The second component is responsible for optimizing the routes by means of solving a (static) VRP instance in each time slice. To this end a GA implementation following Ombuki-Berman et al. paper 20, enhanced with the local memetic optimization 9 is proposed.

\subsection{Time slices}

Following [8] and many other subsequent papers, a working day is split into $n_{t s}$ equal-length time slices and in each time slice a static version the problem (VRP) is solved for the set of currently known customers (requests).

${ }_{140}$ New requests arriving during the current time slice are postponed to its end and optimized in the next algorithm's run (in the next time slice).

Once the calculations allotted for a given time slice are completed the bestfitted chromosome is selected, decoded and the vehicle routes it represents are examined. Roughly speaking, if the time-span of a planned route allows for 145 a "safe time reserve" the vehicle is not moving as it is generally beneficial to wait for another time slice and include newly-arrived requests in the planned solution. Certainly, waiting for too long poses the risk of not being able to extend the route in future time slices by adding new customers as completing the route before the required time $t_{c}$ would not be possible. 
Efficient addressing of the above trade-off is one of the most salient issues in the proposed solution method. To this end the so-called starting delay $(S d, 0 \leq$ $S d \leq 1)$ is defined as the fraction of a working day time $\left(t_{c}-t_{o}\right)$ in which the planned route must be completed. The remaining time, i.e. $(1-S d)\left(t_{c}-t_{o}\right)$, serves as a time reserve for the future route modifications which may possibly be required. Hence if, for a given vehicle, a planned return time to the depot is greater than $S d\left(t_{c}-t_{o}\right)$ the vehicle is moving to the first unserved customer on the assigned route. The customers already visited and the ones to whom the vehicle is already dispatched are removed from the chromosome and called committed. If the currently planned route's time does not exceed $S d\left(t_{c}-t_{o}\right)$ the vehicle is waiting at its current location (either in the depot or at the last customer's) and no customers assigned to it are removed from that chromosome.

The new population (in the next time slice) consists of the copies of the best chromosome from the previous time slice extended by adding the newlyarrived demands (customers). Each new customer (that appeared during the previous time slice) is placed at the end of a route of a randomly chosen vehicle, independently for each chromosome.

Figure 1 outlines the schematic workflow of the algorithm within each time slice.

\subsection{Chromosome representation and initial population}

We use chromosome representation proposed in 20] in which chromosomes are integer vectors of variable length. Positive genes represent clients' identifiers and negative ones correspond to vehicles. Each chromosome contains all and only those identifiers of customers' requests which had already been received (are known to the system) but have not yet been served or committed to any vehicle.

A solution is decoded from a chromosome by scanning it from left to right. Each customer is assigned to a vehicle represented by the last negative number located before that customer's id. In other words, all customers between the two negative identifiers are allocated to the vehicle represented by the first of 


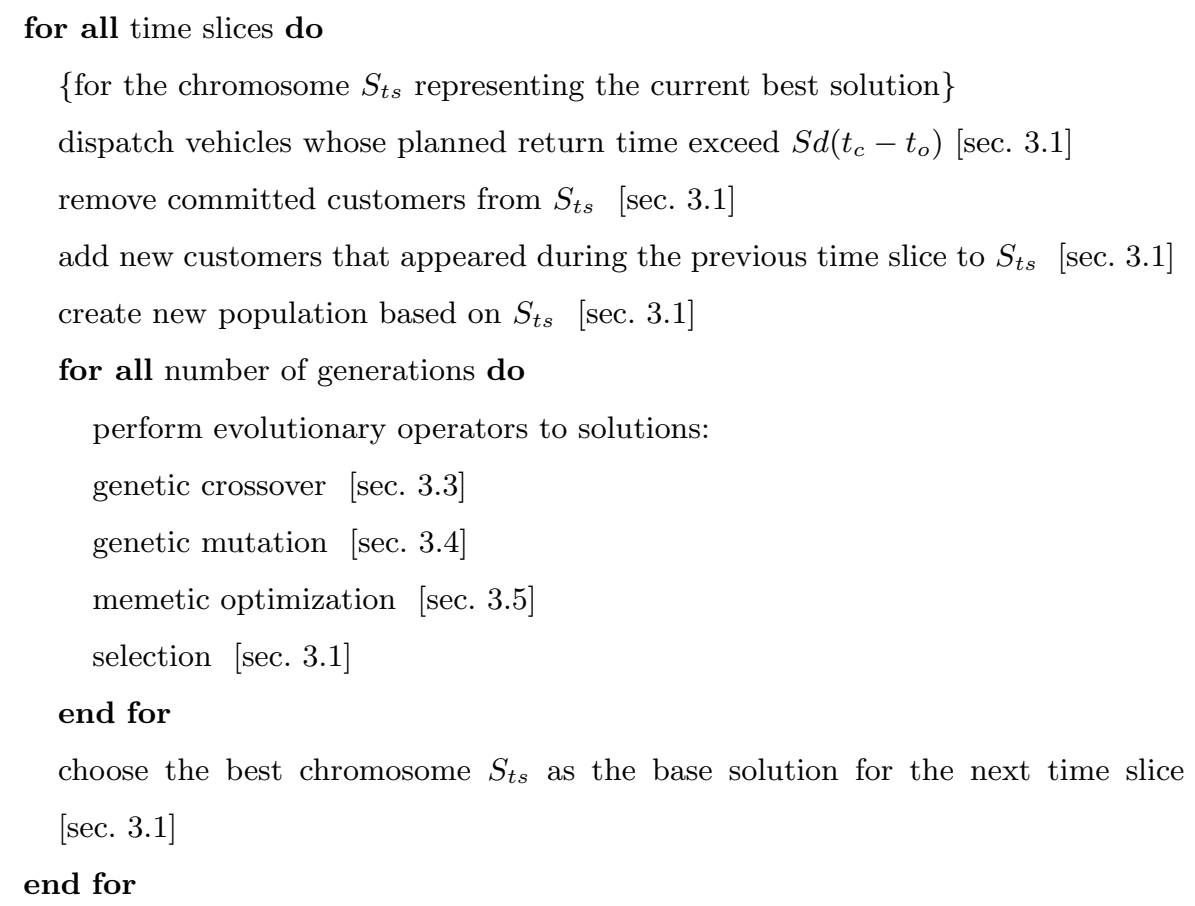

Figure 1: Actions performed during each time slice.

180

185

chromosome begins with a positive number (customer's id) the new vehicle is added as the first element in the chromosome.

Thanks to this automatic repairing procedure (which is applied before each evaluation or potential modification of a chromosome) there is no possibility that a chromosome would represent incorrect solution in any phase of the algorithm.

An example of chromosome representation and its decoding is presented in Figure 2, A chromosome is encoding 8 customers' demands (positive identifiers from 1 to 8 ) and 4 vehicles (negative identifiers from -1 to -4 ). Since the 


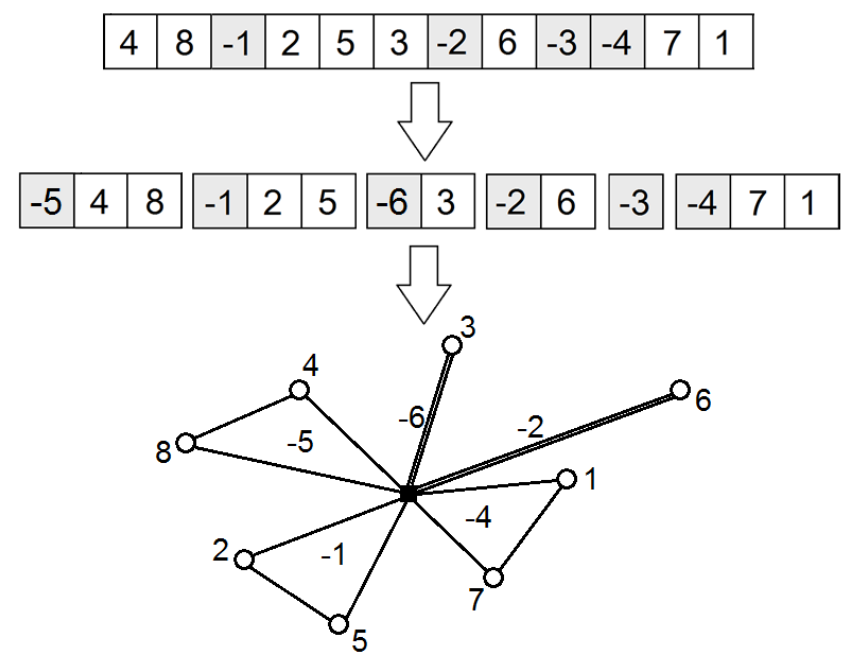

Figure 2: Chromosome representation and solution decoding.

first element of a chromosome is positive, a new vehicle (with id. -5) is added immediately before this element. Orders for customers 4 and 8 are assigned to a planned route for this vehicle, assuming that these requests do not violate capacity constraint. Next we move to another vehicle's id., i.e. -1 and the requests assigned to it. Suppose that the first two customers (2 and 5 ) do not violate the capacity constraint, but adding the third one (3) would have caused exceeding this limit. In such a case a new vehicle (with id. -6) is inserted just before the customer 3 , so as to serve this customer as (at the moment) the sole element of its route. The remaining part of a chromosome is decoded in a similar way based on the above mentioned principles. It should be noted that there is a possibility that a vehicle may have no orders assigned, as vehicle -3 in our example.

The initial population is composed of chromosomes that represent all demands known at the beginning of a day, i.e. those which arrived after the cut-off time of the previous day (see benchmark sets' description in sec. 4.1 for a detailed explanation of this parameter's meaning). Each chromosome is defined as a random permutation of these requests. Hence, at the start of the 
algorithm all chromosomes have the same length and contain the same numbers (customers' identifiers). Please recall that before the first GA operation (the crossover) all chromosomes will be verified against fulfilling the capacity and feasibility rules described above, and "fixed" (if necessary) by adding an appropriate number of vehicles.

\subsection{Crossover}

Our first attempt was to apply crossover operators proposed by Chen et al. [30, but the results were not satisfactory. Therefore, after some limited number of trials we decided to implement the Best-Cost Route Crossover scheme proposed in 20, which yielded much more competitive solutions.

In the Best-Cost Route Crossover two parents $p 1$ and $p 2$ are selected from the population and fixed by applying the repairing procedure. Then two routes $r 1$ and $r 2$ are randomly selected from $p 1$ and $p 2$, respectively and the customers assigned to $r 1$ are deleted in $p 2$ likewise those belonging to route $r 2$ are extracted from $p 1$. Next, the customers that have been removed are reinserted, one after the other, to their parent chromosomes at the locally optimal positions, i.e. in a way that locally minimizes the overall length of the entire set of routes of all vehicles. Please note that even though this type of crossover is quite expensive since it requires examining every position in a chromosome

230 as a potential destination for the reinserted customer, the running time of the algorithm on a single PC does not exceed 10 minutes (24 seconds per time slice, in average). An example of crossover is presented in Figure 3 .

\subsection{Mutation}

Mutation is applied with some probability and mutation operator, for a given chromosome, can take one of the three following forms:

- inversion - two points in a chromosome are randomly selected and all elements between these points are reversed (see Figure 4),

- insertion - a randomly chosen element is removed from a chromosome and then inserted in a randomly selected position, 


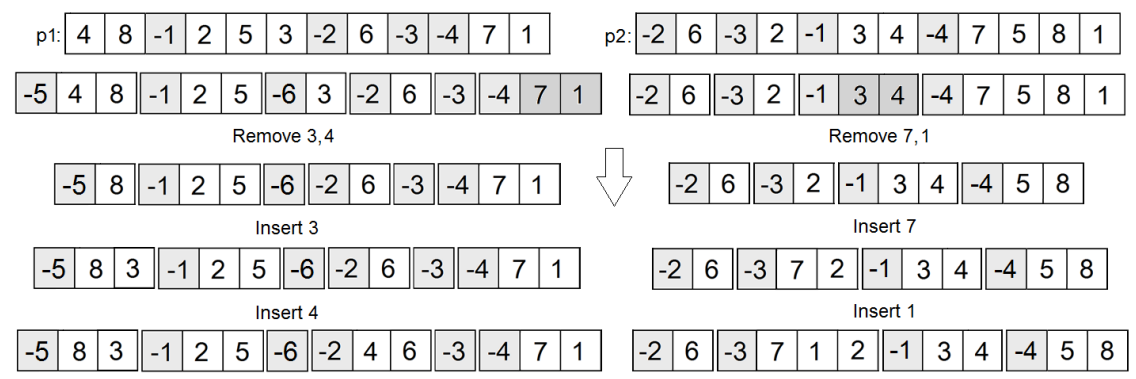

Figure 3: An example of the Best-Cost Route Crossover enhanced with the repairing scheme. First the initial chromosomes are "fixed" based on capacity and feasibility rules. Then the routes of vehicles -4 and -1 are selected in $p_{1}$ and $p_{2}$, respectively and afterwards the respective customers $\left(3,4\right.$ in $p_{1}$ and 7,1 in $\left.p_{2}\right)$ are reinserted in a greedy manner.

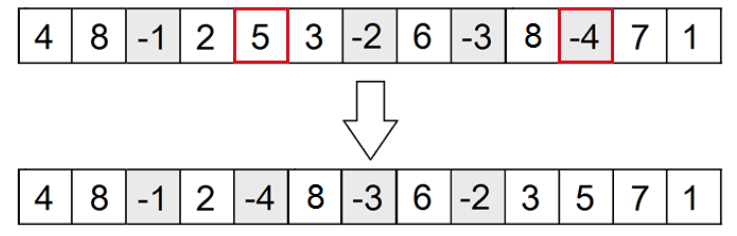

Figure 4: An example of inversion mutation for elements 5 and -4 . The order of genes $[5,3, \ldots,-4]$ is reversed.

- swap - two adjacent elements are exchanged.

A particular form of mutation is selected at random with equal probability of occurrence $\left(=\frac{1}{3}\right)$ of each of the above-listed three versions. It is important to note that in all cases there is no distinction between genes representing vehicles and customers.

\subsection{Memetic optimization}

Memetic optimization is a kind of a local enhancement process which uses problem-related knowledge to improve the solution. Our implementation of memetic optimization adapts the idea of Self-Adaptive Memeplex Individual Learning proposed in 99. The local improvement algorithm is controlled by memes. Each meme serves as a single instruction for local optimization and is 
composed of three elements:

- move operator $T_{i}, i=1, \ldots, 6$,

- acceptance strategy $A$,

- search depth $D$.

A move operator implements one of the following schemes:

$T_{1}$ : a randomly selected customer is removed from a vehicle and reinserted into a newly-added vehicle;

$T_{2}$ : two randomly selected customers from two different vehicles are swapped;

$T_{3}$ : two randomly selected sub-routes from two different vehicles are swapped;

$T_{4}$ : routes of two randomly selected vehicles are merged into one route / vehicle;

$T_{5}$ : a randomly selected customer is removed from a vehicle and reinserted into another vehicle at a random position;

$T_{6}$ : two customers in the same vehicle are randomly selected and the order of all elements located between these customers is reversed.

Each meme uses one of the following two acceptance strategies $A$ :

- first improvement - memetic optimization is stopped after the first improvement is obtained;

- best improvement - memetic move operator is called $D$ times and the best improvement found in these $D$ trials is chosen and applied.

In both above strategies, if $D$ trials of applying meme's move operator did not lead to finding any improvement to the current solution, the chromosome would remain unchanged.

Each chromosome maintains a separate set of $n_{m}$ memes. For each chromosome and for each meme in that chromosome one of the six move operators $T_{i}$ and one of the two acceptance strategies $A$ are randomly and independently chosen with uniform probability (equal to $\frac{1}{6}$ and $\frac{1}{2}$, respectively). Search depth $D$ is common for all memes in all chromosomes. 
The order of memes' application in the memetic improvement phase depends on a synergy matrix $W$. $W$ is $n_{m} \times n_{m}$ matrix unique for each chromosome. Its elements $w_{i j}$ represent the connectivity from meme $m_{i}$ to meme $m_{f^{1}}$. During memetic optimization phase, after applying meme $m_{i}$ the next meme $m_{j}$ is chosen according to the following probability $P_{a}\left(m_{i}, m_{j}\right)$ :

$$
P_{a}\left(m_{i}, m_{j}\right)= \begin{cases}\frac{w_{j j}}{\sum_{\mu=1}^{n_{m}} w_{\mu \mu}} & m_{i}=\emptyset \\ \frac{w_{i j}}{\sum_{\mu=1}^{n_{m}} w_{i \mu}} & \text { otherwise }\end{cases}
$$

Initially matrix $W$ is composed of all ones. After each application of $m_{i}$ followed by application of $m_{j}$ the element $w_{i j}$ is updated according to the following rule:

$$
w_{i j}=\gamma w_{i j}+\frac{c_{j}}{t_{j}}
$$

where $c_{j}$ is the solution improvement caused by application of meme $m_{j}, t_{j}$ is a computational cost of applying meme $m_{j}, \gamma, 0<\gamma<1$ is a discount factor, which specifies the influence of a meme's recent performance over the past. Consequently, the higher the improvement $c_{j}$ and the lower the cost $t_{j}$ of applying meme $m_{j}$ immediately after meme $m_{i}$, the higher the probability of selection of $m_{j}$ as the subsequent meme right after $m_{i}$.

A pseudocode of memetic optimization procedure is shown in Figure 5 . The selection and application of meme $m_{j}$, as well as updating matrix $W$ are repeated until the recently applied meme did not provide any solution improvement $\left(c_{j}=0\right)$ and the exponential ratio between the cumulative improvement and computational cost (of all applied memes) is relatively high $\left(\operatorname{rand}(0,1)>\exp \left(-\frac{t}{c}\right)\right)$. Please observe, that application of meme $m_{j}$ in line 5 denotes repetitive, multiple-time use of its move operator $T$ until the first improvement occurs or the number of trials exceeds $D$, depending on the particular acceptance strategy $A$ associated with meme $m_{j}$. Whenever chromosomes undergo mutation / crossover operation the respective matrix / matrices are also

\footnotetext{
${ }^{1}$ For the sake of clarity of the formulas the connection between matrix $W$ and a chromosome it is assigned to will be omitted. Unless otherwise stated, in all contexts matrix $W$ will refer to the currently considered chromosome.
} 


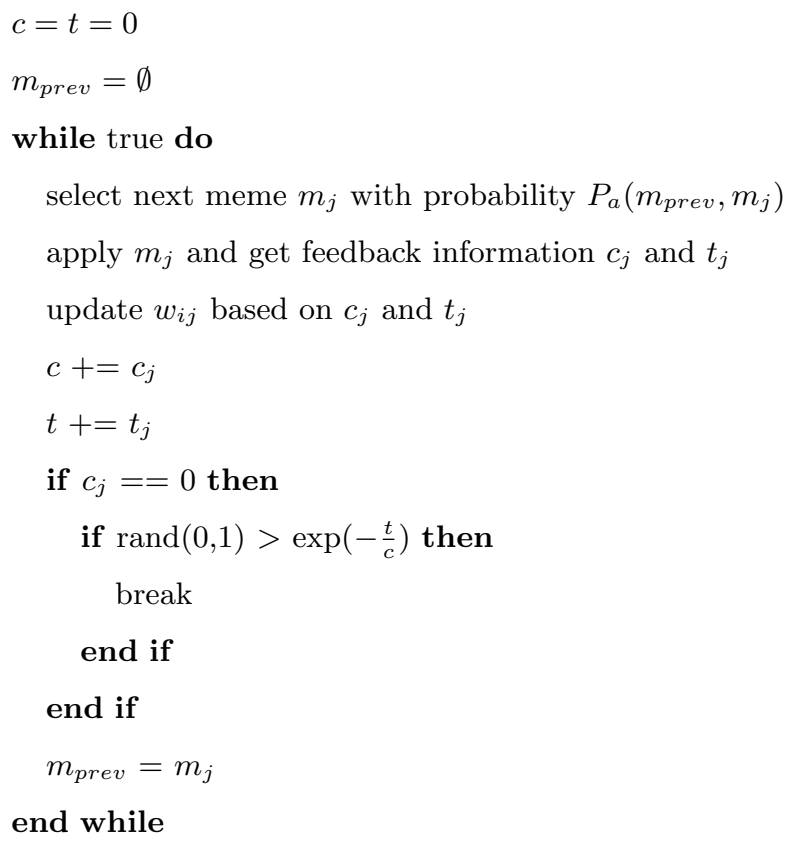

Figure 5: Memetic optimization scheme.

modified. More precisely, during the crossover of chromosomes $a$ and $b$ new matrices $W_{a}$ and $W_{b}$ are created by random exchange of corresponding elements between parents matrices. For each pair of indices $(i, j)$ the element $W_{a}[i, j]$ is swapped with the element $W_{b}[i, j]$ with probability $\frac{1}{2}$.

Mutation of a synergy matrix $W_{a}$ is realized by adding to each element $W_{a}[i, j]$ a small perturbation generated from Gaussian distribution $N\left(0,\left(\frac{\alpha(a)}{100}\right)^{2}\right)$, where $\alpha(a)$ is the average value of all elements in $W_{a}$.

In summary, the role of synergy matrix $W_{a}$ associated with chromosome $a$ is to maintain probability values $w_{i j}$ of applying meme $\left(m_{j}\right)$ immediately after meme $\left(m_{i}\right)$ based on historical gain (high $c_{j}$ and low $t_{j}$ ) of such application. Mutation and crossover of synergy matrices are applied for the same reasons as in the case of operations on chromosomes, i.e. to avoid stagnation in a local minimum and to maintain diversity. 


\section{Experimental setup}

In this section a set of benchmark problems used to evaluate the quality

a day are known for the scheduling system since its starting time and those scheduled in the first half of a day will become known to the solving system in due time.

Two example solutions for the benchmarks $c 50 D$ and $c 100 b D$ are show in 335 Figures 6 and 7, respectively. Please observe very distinct requests distributions and consequently topologies of the routes in these two data sets. This issue is further discussed in Section 5 . 


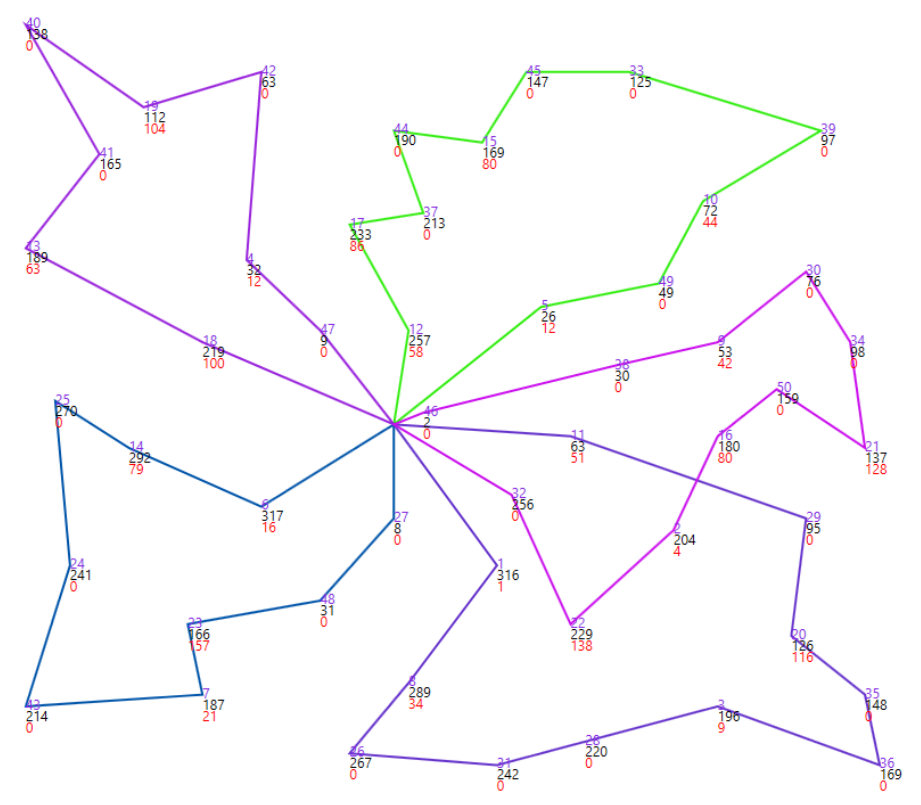

Figure 6: Route networks for instance c50D.

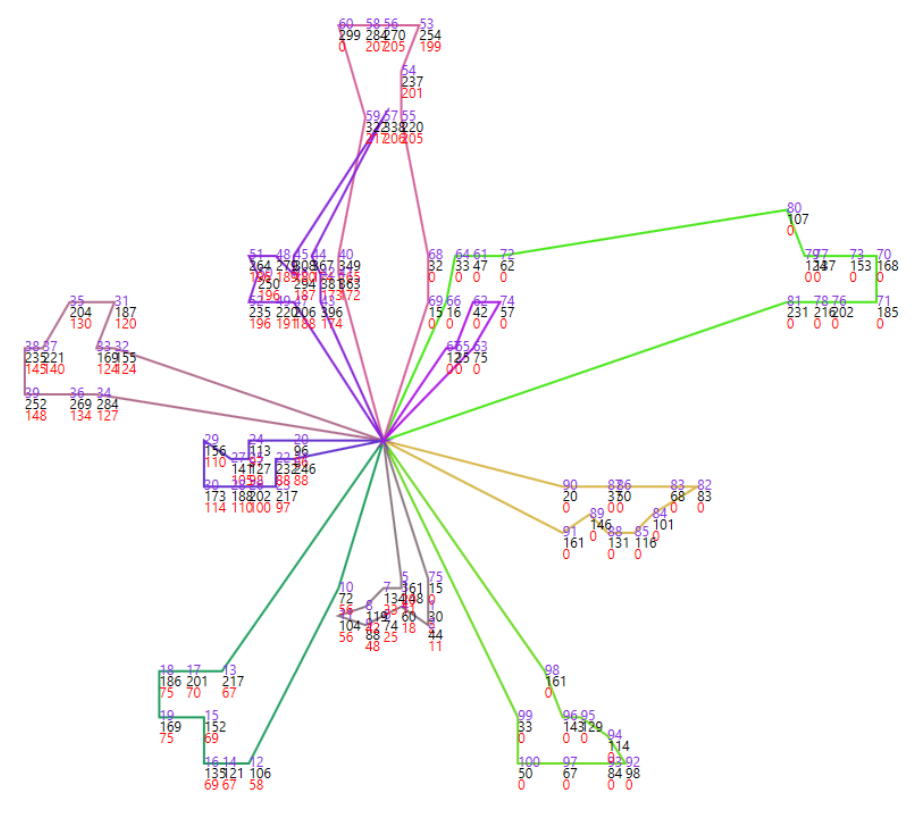

Figure 7: Route networks for instance c100bD. 


\subsection{Parameters' selection}

The parameter which turned out to be the most influential on the solution ness function evaluations constant. In other words, if the population size was increased a relative decrease in the number of generations was imposed. The following pairs of (population, generation) values were tested: $(15,20000),(30,10000)$ 
and $(60,5000)$. The best results were accomplished by a combination $(15,20000)$

- the winner in 13 cases, followed by $(30,10000)$ - which won in 6 cases and by $(60,5000)$ which was the winning selection for two benchmarks. Similarly to crossover rates, the differences among the tested pairs were insignificant, below $2 \%$. The final choice of the main algorithm's parameters is presented in Table 1 .

\begin{tabular}{r|l} 
Parameter & Value \\
\hline cut-off time & 0.5 \\
population size & 15 \\
generation span & 20000 \\
number of time slices & 25 \\
starting delay $(S d)$ & 0.9 \\
crossover rate & 0.7 \\
mutation rate & 0.5 \\
selection type & binary tournament \\
selection pressure & 0.8 \\
number of elite & 2 \\
meme search depth $(D)$ & 100 \\
discount factor $(\gamma)$ & 0.5
\end{tabular}

Table 1: Values of the main steering parameters of the algorithm.

\subsection{Comparative approaches}

In our previous works [21, 22, 25] a new approach to solving VRPDR which utilizes the Two-Phase Multi-Swarm Particle Swarm Optimization method (2MPSO) was proposed. Since the detailed description of the method can be found in the above-cited sources, let us here only briefly describe the underpinning concepts of the 2MPSO algorithm.

In short, in 2MPSO method, similarly to other approaches reported in the 
literature, the working day is divided into some number of time slices and in each of them a currently available instance of the (static) VRP is solved. What accounts for the main difference between 2MPSO and majority of the CI-based approaches described in the literature is that in each time slice the solving process of $2 \mathrm{MPSO}$ is split into two separate phases: a clustering phase (i.e. assignment of requests to vehicles) and optimization phase (i.e. route construction for each of the vehicles). Each of these two above-mentioned subproblems is solved by a separate multi-swarm PSO system. One of the key features of the 2MPSO is effective knowledge transfer between multiple swarms (in-phase) and between swarms used in consecutive time slices.

In the first phase each particle is represented as a real number vector whose elements denote centers of clusters of requests assigned to vehicles. The area of clients' requests is divided among vehicles on the basis of the Euclidean distances from the client's location to the clusters' centers (i.e. a request is assigned to a vehicle which serves the nearest cluster).

In the second phase each particle represents the order of requests assigned to a given vehicle (each cluster/vehicle is solved by a separate PSO instance). The order is obtained by sorting particles' coordinates in the ascending order. The solution assessment in the second phase (in each of the PSO instances) is equal to the length of a route (of a given vehicle) defined by the proposed ordering. The final cost value is equal to the sum of the assessments of the best solutions found by each of the PSO instances.

In 22 the 2MPSO algorithm was extensively compared with several populationbased methods, namely the Ant Colony Optimization [19, evolutionary approach [20], Tabu Search [20] and another, well-established in the literature, PSO-based approach: MEMSO/MAPSO 24, 23, 34, by Khouadija et al. The tests were performed on the same set of benchmarks as those used in this work, described in section 4.1 .

On a general note, the extensive experimental comparison proved an upper hand of the $2 \mathrm{MPSO}$ approach, which for comparable $\left(\approx 10^{7}\right)$ number of fitness function evaluations was capable of finding new best literature solutions in 11 
out of 21 test instances. In terms of the average results the 2MPSO outperformed the competitive methods by at least $1.5 \%$. For the sake of clarity of the presentation the experimental results and conclusions drawn in 22 are not repeated in detail here. Due to the superiority of 2MPSO over all the above-listed metaheuristic approaches we will use this method as a reference point to our memetic VRPDR algorithm (M-VRPDR).

\section{Experimental results}

One of the most universal complexity measures of population-based methods is the number of fitness function evaluations (FFE) of the candidate solutions. This measure allows for making (rough) comparisons between various GA/EA approaches as well as Particle Swarm Optimization or Tabu Search methods. In our method the fitness evaluation is performed in three parts of the algorithm: in crossover operation (in order to find the best position in a chromosome for insertion of removed customer), in selection (so as to choose better-fitted chromosomes for the next generation) and in memetic optimization (to check how the meme operation affects the cumulative length of the vehicle routes). In all baseline numerical results presented in this section the number of FFE in a single algorithm's run is close to (but never exceeds) $10^{7}$, which is the value used to calculate the main results obtained in 22 for other methods.

Results of experiments with steering parameters set according to Table 1 are presented in Tables 2, 3 and 5. The first table shows the best result and the average result out of 50 independent trials for each of the 21 benchmark sets of our M-VRPDR approach and the 2MPSO method (in both cases with a budget of $10^{7} \mathrm{FFE}$ ). In terms of the ability to find the best possible solution (Min category comparison) M-VRPDR outperforms 2MPSO by a large margin - winning in 17 benchmarks and losing in 4 cases. In particular, M-VRPDR proved to be better suited for all Taillard's benchmarks.

When comparing the average performance, which in practice is usually more desirable, as it addresses the issue of repeatability and stability of results the 
advantage of M-VRPDR is not so prevailing, though the method yielded better averages in 14 test sets, losing in the remaining 7 . The memetic approach again showed its upper-hand in the case of Taillard's instances. The difference in average results between the two methods is statistically significant for $p-$ value $=0.007$ for 1 -tailed t-test for averages and $p-$ value $=0.015$ for 2-tailed t-test.

Table 3 compares the best results of M-VRPDR with those found in the literature (including the results of 2MPSO). Both results are attained with the same number of FFE. Our method found the new best literature results in 14 cases.

A more detailed results, including the worst outcomes and standard deviations of the 50 tests with M-VRPDR for each of the benchmarks, as well as the exemplar convergence graphs for the sets c100bd and tai150aD are presented in Appendix A.

\subsection{Hard cases for the $M$-VRPDR}

Based on the average results presented in Table 2 it can be noticed that the algorithm does not manage equally well for all benchmarks. The worst average results compared to $2 \mathrm{MPSO}$ were achieved for instances c100bD and c120D. In these two problems distributions of clients' locations have the form of highly-spatial clusters composed of uniformly distributed request sizes. Figures 8 and 9 show the routes proposed by our algorithm for c100bD and c120D benchmarks. In both presented solutions there exist routes which include requests from two different clustered groups of clients which elongate the overall solution (if these clusters were served by different vehicles the solutions might be shorter). According to our intuition such a behavior can be attributed to the vehicles' starting delay. The imposed condition that a vehicle can move to a customer only if a planned return time to the depot exceeds $90 \%$ of the working day's span causes the planned routes to be relatively long so as to extend routing time beyond the assumed part of the working day. In the case of clustered clients it might potentially be more efficient to serve only one cluster 


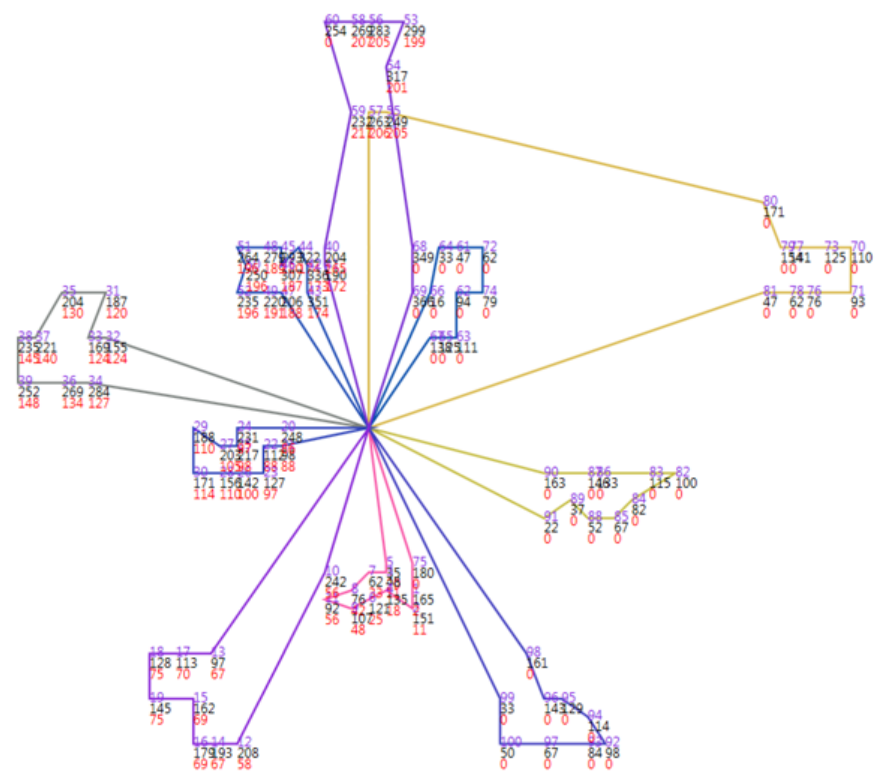

Figure 8: Route networks for instance c100bD.

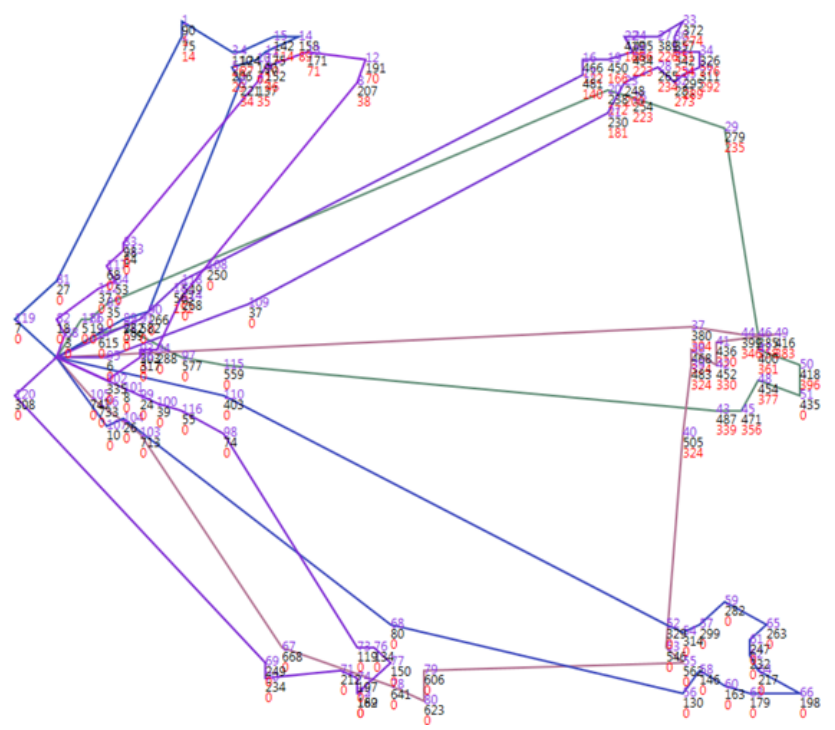

Figure 9: Route networks for instance c120D. 


\begin{tabular}{|r||r|r||r|r|}
\hline \multicolumn{1}{|c||}{} & M-VRPDR $\left(10^{7}\right)$ & \multicolumn{2}{|c|}{2 MPSO $\left(10^{7}\right)$} \\
\hline & Min & Avg & Min & Avg \\
\hline c50D & $\mathbf{5 2 4 . 6 1}$ & $\mathbf{5 4 8 . 1 0}$ & 552.47 & 591.29 \\
\hline c75D & $\mathbf{8 5 2 . 9 5}$ & $\mathbf{8 8 5 . 0 0}$ & 878.93 & 904.12 \\
\hline c100D & $\mathbf{8 6 0 . 5 6}$ & $\mathbf{9 1 3 . 8 1}$ & 874.20 & 920.84 \\
\hline c100bD & 820.92 & 864.03 & $\mathbf{8 1 9 . 5 6}$ & $\mathbf{8 3 9 . 0 1}$ \\
\hline c120D & 1189.06 & 1295.31 & $\mathbf{1 0 5 6 . 2 8}$ & $\mathbf{1 1 3 1 . 3 0}$ \\
\hline c150D & $\mathbf{1 0 8 3 . 7 9}$ & 1142.24 & 1096.53 & $\mathbf{1 1 3 1 . 7 8}$ \\
\hline c199D & 1377.26 & 1466.30 & $\mathbf{1 3 6 2 . 8 4}$ & $\mathbf{1 4 1 5 . 5 8}$ \\
\hline f71D & $\mathbf{2 6 0 . 1 7}$ & 297.61 & 282.69 & $\mathbf{2 9 1 . 6 1}$ \\
\hline f134D & 11815.95 & 12299.59 & $\mathbf{1 1 7 5 5 . 5 8}$ & $\mathbf{1 2 0 3 8 . 2 8}$ \\
\hline tai75aD & $\mathbf{1 6 5 6 . 1 2}$ & $\mathbf{1 7 0 5 . 7 6}$ & 1682.90 & 1766.68 \\
\hline tai75bD & $\mathbf{1 3 5 0 . 2 8}$ & $\mathbf{1 3 7 8 . 4 7}$ & 1404.29 & 1447.30 \\
\hline tai75cD & $\mathbf{1 3 5 5 . 4 1}$ & $\mathbf{1 4 2 7 . 7 4}$ & 1406.59 & 1498.18 \\
\hline tai75dD & $\mathbf{1 3 7 6 . 4 2}$ & $\mathbf{1 4 1 4 . 9 3}$ & 1415.79 & 1439.78 \\
\hline tai100aD & $\mathbf{2 0 9 3 . 6 3}$ & $\mathbf{2 1 6 8 . 8 7}$ & 2155.42 & 2215.44 \\
\hline tai100bD & $\mathbf{1 9 9 0 . 9 9}$ & $\mathbf{2 0 9 7 . 8 6}$ & 2022.13 & 2102.15 \\
\hline tai100cD & $\mathbf{1 4 2 1 . 5 0}$ & $\mathbf{1 4 6 0 . 7 2}$ & 1446.10 & 1493.59 \\
\hline tai100dD & $\mathbf{1 6 3 1 . 6 3}$ & $\mathbf{1 7 1 0 . 4 4}$ & 1690.32 & 1753.17 \\
\hline tai150aD & $\mathbf{3 2 2 6 . 5 1}$ & $\mathbf{3 3 5 4 . 0 9}$ & 3298.91 & 3394.92 \\
\hline tai150bD & $\mathbf{2 8 4 7 . 0 8}$ & $\mathbf{2 9 8 0 . 3 3}$ & 2887.88 & 2992.81 \\
\hline tai150cD & $\mathbf{2 4 2 7 . 5 3}$ & 2558.99 & 2462.96 & $\mathbf{2 5 5 1 . 5 8}$ \\
\hline tai150dD & $\mathbf{2 7 3 7 . 3 7}$ & $\mathbf{2 9 0 3 . 6 8}$ & 2886.12 & 2941.66 \\
\hline
\end{tabular}

Table 2: The best and the average results of $M-\operatorname{VRPDR}\left(10^{7}\right)$ and $2 M P S O\left(10^{7}\right)$ for the considered benchmarks. The better of the two results in each category (Min, Avg) is bolded.

of clients per vehicle and return to the depot immediately after, regardless of the time of a day and available capacity left. In order to verify this hypothesis, 50 tests were performed for these two benchmarks with $S d$ times set to 


\begin{tabular}{|c|c|c|c|c|}
\hline & M-VRPDR & Best other & Algorithm & $\# \mathrm{FFE}$ \\
\hline $\mathrm{c} 50 \mathrm{D}$ & 524.61 & 552.47 & $2 \mathrm{MPSO}_{3} \quad 22$ & $10^{7}$ \\
\hline c75D & 866.45 & 877.30 & $2 \mathrm{MPSO}_{2} 22$ & $2 \cdot 10^{6}$ \\
\hline $\mathrm{c} 100 \mathrm{D}$ & 873.10 & 874.20 & $2 \mathrm{MPSO}_{3}$ 22] & $10^{8}$ \\
\hline $\mathrm{c} 100 \mathrm{bD}$ & 820.92 & 819.56 & $2 \mathrm{MPSO}_{3} 22$ & $10^{8}$ \\
\hline $\mathrm{c} 120 \mathrm{D}$ & 1199.64 & 1056.28 & $2 \mathrm{MPSO}_{3}$ & $10^{8}$ \\
\hline $\mathrm{c} 150 \mathrm{D}$ & 1083.79 & 1096.53 & $2 \mathrm{MPSO}_{3}, 22$ & $10^{7}$ \\
\hline c199D & 1376.26 & 1362.84 & $2 \mathrm{MPSO}_{3}, 22$ & $10^{8}$ \\
\hline f71D & 275.51 & 278.65 & $2 \mathrm{MPSO}_{3}$ & $10^{5}$ \\
\hline $\mathrm{f} 134 \mathrm{D}$ & 11817.66 & 11755.58 & $2 \mathrm{MPSO}_{3} \quad 22$ & $10^{8}$ \\
\hline tai75aD & 1658.13 & 1682.90 & $2 \mathrm{MPSO}_{3}, 22$ & $10^{8}$ \\
\hline tai75bD & 1351.06 & 1391.74 & $2 \mathrm{MPSO}_{3} 22$ & $2.2 \cdot 10^{7}$ \\
\hline tai75cD & 1388.13 & 1406.27 & TabuSearch 20] & $25 \cdot 30 \mathrm{sec}$ \\
\hline tai75dD & 1395.30 & 1342.26 & $M E M S O \quad 23]$ & $10^{6}$ \\
\hline tai100aD & 2122.92 & 2146.53 & $2 \mathrm{MPSO}_{2}$ & $2.2 \cdot 10^{7}$ \\
\hline tai100bD & 1990.99 & 2022.13 & $2 \mathrm{MPSO}_{3} 22$ & $10^{7}$ \\
\hline tai100cD & 1419.89 & 1446.10 & $2 \mathrm{MPSO}_{3} 22$ & $10^{8}$ \\
\hline tai100dD & 1651.28 & 1685.53 & $2 \mathrm{MPSO}_{2} 22$ & $2.2 \cdot 10^{7}$ \\
\hline tai150aD & 3343.54 & 3253.77 & MEMSO 23] & $10^{6}$ \\
\hline tai150bD & 2957.16 & 2861.91 & $M A P S O$ & $10^{6}$ \\
\hline tai150cD & 2430.91 & 2462.96 & $2 \mathrm{MPSO}_{3}, 22$ & $10^{8}$ \\
\hline tai150dD & 2742.35 & 2844.70 & $2 \mathrm{MPSO}_{2} 22$ & $2.2 \cdot 10^{7}$ \\
\hline
\end{tabular}

Table 3: Comparison of the best M-VRPDR results ( $2^{n d}$ column) with the hitherto bestknown literature solutions ( $3^{\text {rd }}$ column) accomplished by the algorithm presented in the $4^{\text {th }}$ column. Both results are attained with the same number of FFE ( $5^{\text {th }}$ column) - defined in the cited papers. In one case (tai75cD), following [20, instead of the number of FFE, the time measure is used for methods' comparison. For each benchmark the better outcome is bolded. 
$0.0,0.05,0.1,0.15, \ldots, 0.85$ hoping that the possibility to start the route earlier would alleviate the problem of ineffective (packed close to the capacity limit) routes. Unfortunately, all these values appeared to be weaker choices than 0.9 - the setting used in the main experiment. The main reason for that is a large number of trucks which are at the algorithm's disposal (in all tested benchmarks this limit is set to 50, which is well beyond the practical needs). Having sufficient number of spare vehicles there is no real need for any truck to optimize its route in a way which would allow the vehicle to make two tours within a day. Consequently, the trucks were leaving depot earlier than in the main experiment but at the same time were much more "careless" about the route optimization, having enough time and capacity to extend the solution in different ways. It seems that the case of densely clustered customers' distributions requires special attention and we have put this issue on the top of our priority list.

Furthermore, visibly worse results were also obtained for benchmarks c150D and c199D. While we could not trace any specific attributes of these sets other than their size, we hypothesized that the number of iterations might have been too small to allow the convergence to a good solution due to the higher (than in other cases) number of customers. In order to verify this claim, we performed additional tests for all four "resistant" benchmarks: c100bD, c120D, c150D, c199D with doubled number of iterations. The results are presented in Table 4

495 In the case of bigger benchmark sets (c150D and c199D) the results improved by about $2.5 \%$ which seems to confirm our presumption. For the densely clustered problems, however, the improvement is meaningless, about $1 \%$.

\section{Saliency of the memetic component}

While encouraging experimental results, in particular the ability to find new 500 best literature results for a bunch of popular and widely-recognizable benchmarks, give prospects for possible applicability and further development of the M-VRPDR, from the research point of view the most pertinent question is related to the reasons of such a promising behavior. 


\begin{tabular}{|r||r|r||rr|}
\hline \multicolumn{1}{|c||}{} & \multicolumn{2}{c||}{$\begin{array}{c}\text { M-VRPDR with } \\
\text { doubled iterations }\end{array}$} & \multicolumn{2}{c|}{ M-VRPDR } \\
\hline & Min & Avg & Min & Avg \\
\hline c100bD & 819.56 & 855.74 & 820.92 & 864.03 \\
\hline c120D & 1192.35 & 1282.27 & 1189.06 & 1295.31 \\
\hline c150D & 1071.78 & 1112.12 & 1083.79 & 1142.24 \\
\hline c199D & 1365.26 & 1430.52 & 1377.26 & 1466.30 \\
\hline
\end{tabular}

Table 4: Result of the algorithm with doubled numbers of iterations for 4 benchmarks for which M-VRPDR obtained the worst results.

Based on some number of preliminary tests it turned out that in the parametriza-

tion layer the crucial variable is the starting delay parameter, whose proper setting is indispensable for efficient solving of VRPDR. In order to deeper investigate this issue additional tests were performed aiming at comparison of the "pure" genetic approach (i.e. without memetic optimization) without considering the starting delay parameter, the pure genetic approach with starting delay value set to 0.9 (as in the main experiment) and the M-VRPDR. The lack of memetic optimization phase in both purely genetic implementations was compensated by the increased number of iterations, so as to maintain approximately the same number of FFE.

Table 5 presents comparison of results obtained by M-VRPDR and the two genetic approaches (with and without considering the $S d$ parameter) based on 50 independent runs in each case. The importance of both tested factors (starting delay parameter and memetic optimization) is clearly supported by the results. The purely GA approach, without taking into account $S d$ factor, was not a competitive approach for any of the considered benchmarks. In average it yielded the solution $11.18 \%$ longer than those of the same GA implementation which relied on the starting delay. This enhanced GA approach incidentally appeared to be stronger than M-VRPDR in terms of best-found solution, but in a more systematic measure, by means of the average solution length, it lost 


\begin{tabular}{|r||r|r||r|r||r|r|}
\hline \multicolumn{1}{|c||}{} & \multicolumn{2}{c||}{ GA-Sd } & \multicolumn{2}{c||}{ GA + Ad } & \multicolumn{2}{c|}{ M-VRPDR } \\
\hline & Min & Avg & Min & Avg & Min & Avg \\
\hline c50D & 598.86 & 634.86 & 527.17 & 566.25 & $\mathbf{5 2 4 . 6 1}$ & $\mathbf{5 4 8 . 1 0}$ \\
\hline c75D & 984.83 & 1059.16 & 865.70 & 898.40 & $\mathbf{8 5 2 . 9 5}$ & $\mathbf{8 8 5 . 0 0}$ \\
\hline c100D & 1004.36 & 1061.87 & 868.94 & 940.20 & $\mathbf{8 6 0 . 5 6}$ & $\mathbf{9 1 3 . 8 1}$ \\
\hline c100bD & 880.19 & 996.82 & 824.94 & 884.56 & $\mathbf{8 2 0 . 9 2}$ & $\mathbf{8 6 4 . 0 3}$ \\
\hline c120D & 1282.32 & 1474.45 & 1222.00 & 1421.14 & $\mathbf{1 1 8 9 . 0 6}$ & $\mathbf{1 2 9 5 . 3 1}$ \\
\hline c150D & 1377.09 & 1480.56 & 1089.41 & 1176.25 & $\mathbf{1 0 8 3 . 7 9}$ & $\mathbf{1 1 4 2 . 2 4}$ \\
\hline c199D & 1762.94 & 1888.07 & 1436.00 & 1506.24 & $\mathbf{1 3 7 7 . 2 6}$ & $\mathbf{1 4 6 6 . 3 0}$ \\
\hline f71D & 279.02 & 304.48 & 274.02 & 304.24 & $\mathbf{2 6 0 . 1 7}$ & $\mathbf{2 9 7 . 6 1}$ \\
\hline f134D & 15480.35 & 16309.51 & $\mathbf{1 1 7 9 5 . 5 5}$ & 12711.37 & 11815.95 & $\mathbf{1 2 2 9 9 . 5 9}$ \\
\hline tai75aD & 1758.56 & 1866.33 & 1668.75 & 1735.95 & $\mathbf{1 6 5 6 . 1 2}$ & $\mathbf{1 7 0 5 . 7 6}$ \\
\hline tai75bD & 1480.91 & 1603.10 & 1352.04 & 1382.08 & $\mathbf{1 3 5 0 . 2 8}$ & $\mathbf{1 3 7 8 . 4 7}$ \\
\hline tai75cD & 1516.50 & 1604.15 & 1372.42 & 1456.85 & $\mathbf{1 3 5 5 . 4 1}$ & $\mathbf{1 4 2 7 . 7 4}$ \\
\hline tai75dD & 1456.45 & 1561.61 & 1393.19 & 1424.66 & $\mathbf{1 3 7 6 . 4 2}$ & $\mathbf{1 4 1 4 . 9 3}$ \\
\hline tai100aD & 2185.52 & 2377.83 & 2103.70 & 2193.21 & $\mathbf{2 0 9 3 . 6 3}$ & $\mathbf{2 1 6 8 . 8 7}$ \\
\hline tai100bD & 2136.29 & 2321.65 & 2049.49 & 2141.61 & $\mathbf{1 9 9 0 . 9 9}$ & $\mathbf{2 0 9 7 . 8 6}$ \\
\hline tai100cD & 1564.66 & 1670.13 & 1425.17 & 1475.23 & $\mathbf{1 4 2 1 . 5 0}$ & $\mathbf{1 4 6 0 . 7 2}$ \\
\hline tai100dD & 1801.75 & 1943.67 & 1661.63 & 1742.45 & $\mathbf{1 6 3 1 . 6 3}$ & $\mathbf{1 7 1 0 . 4 4}$ \\
\hline tai150aD & 3541.84 & 3858.63 & 3241.59 & 3409.37 & $\mathbf{3 2 2 6 . 5 1}$ & $\mathbf{3 3 5 4 . 0 9}$ \\
\hline tai150bD & 3109.13 & 3469.71 & $\mathbf{2 8 4 5 . 4 6}$ & 3076.60 & 2847.08 & $\mathbf{2 9 8 0 . 3 3}$ \\
\hline tai150cD & 2738.08 & 2989.05 & 2442.11 & 2643.10 & $\mathbf{2 4 2 7 . 5 3}$ & $\mathbf{2 5 5 8 . 9 9}$ \\
\hline tai150dD & 3055.80 & 3238.90 & 2810.29 & 2958.12 & $\mathbf{2 7 3 7 . 3 7}$ & $\mathbf{2 9 0 3 . 6 8}$ \\
\hline & & & & & & \\
\hline
\end{tabular}

Table 5: Comparison of results obtained by M-VRPDR and the two genetic approaches (with and without considering the $S d$ parameter, denoted by GA+Sd and GA-Sd, respectively) based on 50 independent runs with a budget of $10^{7} \mathrm{FFE}$ in each case. Best results are bolded.

to M-VRPDR in all cases, with the average margin of $2.47 \%$. 


\section{Summary and conclusions}

This paper presents a memetic approach to solving the Vehicle Routing Problem with Dynamic Requests. The algorithm was tested on a well-established set of benchmarks and proved to be an effective and reliable method, capable of finding 13 new best-known results out of 21 tested problems, using the same numbers of fitness function evaluations. It is worth underlying that, except for some parameter tuning, the method was not optimized for solving this particular set of benchmarks. Furthermore, the proposed algorithm can, in principle, be applied to solving other VRPDR benchmarks with no specific adjustments as the selected parameters seem to be universally useful (though certainly not optimal in strict sense).

Our algorithm relies on problem encoding previously introduced in [20] and adopts memetic optimization scheme proposed in [9], however, both these factors are combined in a novel manner as parts of the newly-designed system and applied to the problem other that those considered in the source papers.

540 Furthermore, while memetic optimization is definitely an important part of the overall solution method, the paramount feature is the starting delay parameter which heuristically administers the dispatching times of the vehicles.

Additionally, a new way of knowledge transfer between consecutive partial solutions defined during the day (in subsequent time-slices) is introduced in this study and proved to be effective when combined with the proposed extensive mutation operator which prevents the system from premature convergence or stagnation.

Our current research focus is on development of a meta-heuristic procedure, which - based on the analysis of particular data set (spatial and volume distributions of requests, their skewness and approximate number of clusters) would autonomously adjust the parameters of the method so as to increase its efficacy in solving a given benchmark set. Such a meta-heuristic system can, for instance, be implemented as a one-layer feed-forward network and trained on an ensemble of benchmarks, taking the statistical parameters of the data set as in- 

appeared to be the most demanding for the current system implementation. We hope that due to intrinsic nonlinearity of neural networks' approximation the proposed meta-approach will further strengthen the system's performance.

\section{Acknowledgment}

The research was financed by the National Science Centre in Poland, grant number DEC-2012/07/B/ST6/01527. The authors would like to thank Michał Okulewicz for his helpful comments.

\section{Appendix A}

The appendix presents statistical characteristics of the baseline M-VRPDR $\left(10^{7}\right)$ results (Table 6) and two exemplar convergence graphs (Figures 10 and 11), respectively for c100bD and tai150aD benchmark sets.

The results presented in Table 6 show that the method is reliable (repeatable), as for all benchmarks the standard deviation is kept within reasonable limits compared to the average solution. The greatest spread of outcomes (in

[1] G. B. Dantzig, R. Ramser, The Truck Dispatching Problem, Management Science 6 (1959) 80-91.

[2] L. M. Gambardella, ric Taillard, G. Agazzi, Macs-vrptw: A multiple ant colony system for vehicle routing problems with time windows, in: New per cent points) can be observed for f71D (4.79\%) and tai150cD (4.09\%), while the most consistent results are obtained in the case of f134D (only 1.46\%) and tai75bD (1.51\%). Overall, the worst solutions (column Max) are within the $11.21 \%$ margin (achieved for tai150aD) from the average results across all benchmarks, but can be as low as $3.01 \%$ for $1134 \mathrm{D}$ or $3.43 \%$ for tai75bD.

\section{References} Ideas in Optimization, McGraw-Hill, 1999, pp. 63-76. 


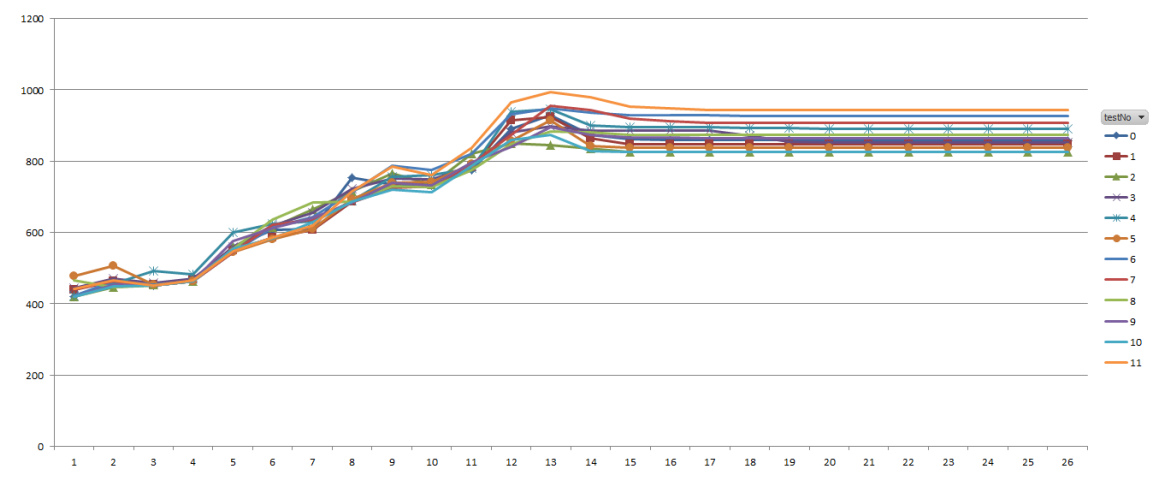

Figure 10: The convergence graphs for 12 independent algorithm's runs for the c100bD benchmark set. The $\mathrm{x}$-axis represents time (time slices) and the y-axis - the length of the currently committed tour. It can be seen from the figure that the experiments are fairly repeatable. After the cut-off time (time slice \#13) only local route optimization takes place.

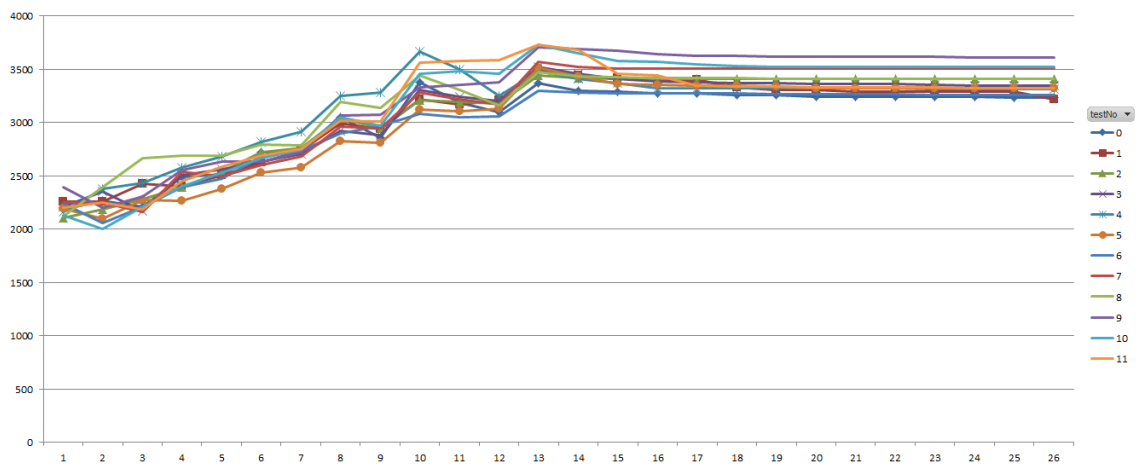

Figure 11: The convergence graphs for 12 independent algorithm's runs for the tai150aD benchmark set. The $\mathrm{x}$-axis represents time (time slices) and the $\mathrm{y}$-axis - the length of the currently committed tour. It can be seen from the figure that the experiments are fairly repeatable. After the cut-off time (time slice \#13) only local route optimization takes place. 


\begin{tabular}{|r||r|r|r|r|r|}
\hline & Min & Avg & Max & St_dev & St_dev/Avg \\
\hline c50D & 524.61 & 548.10 & 582.09 & 17.49 & $3.19 \%$ \\
\hline c75D & 852.95 & 885.00 & 944.83 & 22.14 & $2.50 \%$ \\
\hline c100D & 860.56 & 913.81 & 999.41 & 29.98 & $3.28 \%$ \\
\hline c100bD & 820.92 & 864.03 & 927.51 & 25.93 & $3.00 \%$ \\
\hline c120D & 1189.06 & 1295.31 & 1407.31 & 49.67 & $3.83 \%$ \\
\hline c150D & 1083.79 & 1142.24 & 1234.75 & 38.18 & $3.34 \%$ \\
\hline c199D & 1377.26 & 1466.30 & 1596.62 & 45.08 & $3.07 \%$ \\
\hline f71D & 260.17 & 297.61 & 324.61 & 14.25 & $4.79 \%$ \\
\hline f134D & 11815.95 & 12299.59 & 12669.69 & 180.06 & $1.46 \%$ \\
\hline tai75aD & 1656.12 & 1705.76 & 1797.22 & 34.22 & $2.01 \%$ \\
\hline tai75bD & 1350.28 & 1378.47 & 1425.69 & 20.88 & $1.51 \%$ \\
\hline tai75cD & 1355.41 & 1427.74 & 1538.73 & 37.90 & $2.65 \%$ \\
\hline tai75dD & 1376.42 & 1414.93 & 1481.04 & 24.23 & $1.71 \%$ \\
\hline tai100aD & 2093.63 & 2168.87 & 2357.59 & 53.48 & $2.47 \%$ \\
\hline tai100bD & 1990.99 & 2097.86 & 2195.63 & 55.92 & $2.67 \%$ \\
\hline tai100cD & 1421.50 & 1460.72 & 1564.04 & 31.02 & $2.12 \%$ \\
\hline tai100dD & 1631.63 & 1710.44 & 1820.74 & 49.64 & $2.90 \%$ \\
\hline tai150aD & 3226.51 & 3354.09 & 3730.07 & 106.00 & $3.16 \%$ \\
\hline tai150bD & 2847.08 & 2980.33 & 3134.90 & 74.73 & $2.51 \%$ \\
\hline tai150cD & 2427.53 & 2558.99 & 2828.03 & 104.55 & $4.09 \%$ \\
\hline tai150dD & 2737.37 & 2903.68 & 3063.57 & 67.63 & $2.33 \%$ \\
\hline
\end{tabular}

Table 6: Detailed results of the M-VRPDR method for all 21 benchmarks with a budget of $10^{7} \mathrm{FFE}$ in each test.

[3] A. H. Michel Gendreau, G. Laporte, A tabu search heuristic for the vehicle routing problem, Management Science 40 (10) (1994) 276-1290.

[4] Y. Wu, P. Ji, T. Wang, An empirical study of a pure genetic algorithm to solve the capacitated vehicle routing problem, ICIC Express Letters 2 
(2008) 41-45.

[5] I. H. Osman, Metastrategy simulated annealing and tabu search algorithms for the vehicle routing problem, Annals of Operations Research 41 (4) (1993) 421-451.

[6] J. Mańdziuk, C. Nejman, UCT-based approach to Capacitated Vehicle Routing Problem, in: Artificial Intelligence and Soft Computing, Vol. 9120 of Lecture Notes in Computer Science, Springer Berlin Heidelberg, 2015, pp. 679-690.

[7] N. Wilson, N. Colvin, Computer control of the rochester dial-a-ride system, Tech. Rep. R77-31, Departament of Civil Engineering, Massachusetts Institute of Technology, Cambridge, Massachusetts.

[8] P. Kilby, P. Prosser, P. Shaw, Dynamic VRPs: A Study of Scenarios, accessed: 2015-09-01 (1998).

URL http://www.cs.strath .ac .uk/ apes/apereports.html

[9] X. Chen, L. Feng, Y. Ong, A self-adaptive memeplexes robust search scheme for solving stochastic demands vehicle routing problem, Int. J. Systems Science 43 (7) (2012) 1347-1366.

[10] L. Wen, B. atay, R. Eglese, Finding a minimum cost path between a pair of nodes in a time-varying road network with a congestion charge, European Journal of Operational Research 236 (3) (2014) 915 - 923.

[11] J. Mańdziuk, M. Świechowski, UCT method in stochastic transportation problems, accessed: 2015-09-01 (Unpublished results). URL http://www . mini .pw . edu .pl/ mandziuk/WORK/UCT-DVRP.pdf

[12] D. Tas, M. Gendreau, N. Dellaert, T. van Woensel, A. de Kok, Vehicle routing with soft time windows and stochastic travel times: A column generation and branch-and-price solution approach, European Journal of Operational Research 236 (3) (2014) 789 - 799. 
[13] D. Cattaruzza, N. Absi, D. Feillet, T. Vidal, A memetic algorithm for the multi trip vehicle routing problem, European Journal of Operational Research 236 (3) (2014) $833-848$.

[14] M. M. Abdulkader, Y. Gajpal, T. Y. ElMekkawy, Hybridized ant colony algorithm for the multi compartment vehicle routing problem, Applied Soft Computing 37 (2015) $196-203$.

[15] K. Ghoseiri, S. F. Ghannadpour, Multi-objective vehicle routing problem with time windows using goal programming and genetic algorithm, Appl. Soft Comput. 10 (4) (2010) 1096-1107.

[16] R. Masson, S. Ropke, F. Lehud, O. Pton, A branch-and-cut-and-price approach for the pickup and delivery problem with shuttle routes, European Journal of Operational Research 236 (3) (2014) $849-862$.

[17] P. Toth, D. Vigo, Special issue on vehicle routing and distribution logistics, European Journal of Operational Research 236 (3) (2014) IFC -.

[18] B. Eksioglu, A. V. Vural, A. Reisman, The vehicle routing problem: a taxonomic review, Computers \& Industrial Engineering 57 (4) (2009) 1472 $-1483$.

[19] R. Montemanni, L. Gambardella, A. Rizzoli, A. Donati, A new algorithm for a dynamic vehicle routing problem based on ant colony system, Journal of Combinatorial Optimization 10 (2005) 327-343.

[20] F. T. Hanshar, B. M. Ombuki-Berman, Dynamic vehicle routing using genetic algorithms, Applied Intelligence 27 (1) (2007) 89-99.

[21] M. Okulewicz, J. Mańdziuk, Application of Particle Swarm Optimization Algorithm to Dynamic Vehicle Routing Problem, in: Artificial Intelligence and Soft Computing, Vol. 7895 of Lecture Notes in Computer Science, Springer Berlin Heidelberg, 2013, pp. 547-558. 
[22] M. Okulewicz, J. Mańdziuk, A two-phase multi-swarm algorithm for solving dynamic vehicle routing problem, accessed: 2015-09-01 (Unpublished results).

URL http://www.mini.pw.edu.pl/ mandziuk/WORK/2MPSO.pdf

[23] M. R. Khouadjia, E.-G. Talbi, L. Jourdan, B. Sarasola, E. Alba, Multienvironmental cooperative parallel metaheuristics for solving dynamic optimization problems, Journal of Supercomputing 63 (3) (2013) 836-853.

[24] M. R. Khouadjia, B. Sarasola, E. Alba, L. Jourdan, E.-G. Talbi, A comparative study between dynamic adapted PSO and VNS for the vehicle routing problem with dynamic requests, Applied Soft Computing 12 (4) (2012) 1426-1439.

[25] J. Mańdziuk, S. Zadrożny, K. Walędzik, M. Okulewicz, M. Świechowski, Adaptive metaheuristic methods in dynamically changing environments, accessed: 2015-09-01 (2015).

URL http://www.mini.pw.edu.pl/ mandziuk/dynamic/

[26] Y. S. Ong, M. H. Lim, X. S. Chen, Research frontier: Memetic computation - past, present \& future, IEEE Computational Intelligence Magazine 5 (2) (2010) 24-36.

[27] X. S. Chen, Y. S. Ong, M. H. Lim, K. C. Tan, A multi-facet survey on memetic computation, IEEE Transactions on Evolutionary Computation 15 (5) (2011) 591-607.

[28] F. Neri, C. Cotta, Memetic algorithms and memetic computing optimization: A literature review, Swarm and Evolutionary Computation 2 (2012) $1-14$.

[29] A. K. Raymond Kwan, A. Wren, Evolutionary driver scheduling with relief chains, Evolutionary Computation 9 (4) (2001) 445-460. 
[30] X. Chen, Y. Ong, M. H. Lim, Cooperating memes for vehicle routing problems, International Journal of Innovative Computing, Information and Control 7 (2011) 1-10.

[31] N. Christofides, J. E. Beasley, The period routing problem, Networks 14 (2) (1984) 237-256.

[32] M. L. Fisher, R. Jaikumar, A generalized assignment heuristic for vehicle routing, Networks 11 (2) (1981) 109-124.

[33] É. D. Taillard, Parallel iterative search methods for vehicle routing problems, Networks 23 (8) (1993) 661-673.

[34] M. R. Khouadjia, E. Alba, L. Jourdan, E.-G. Talbi, Multi-Swarm Optimization for Dynamic Combinatorial Problems: A Case Study on Dynamic Vehicle Routing Problem, in: Swarm Intelligence, Vol. 6234 of Lecture Notes in Computer Science, Springer, Berlin / Heidelberg, 2010, pp. 227-238. 


\section{Vitae}

Jacek Mańdziuk PhD, DSc. is Associate Professor

680

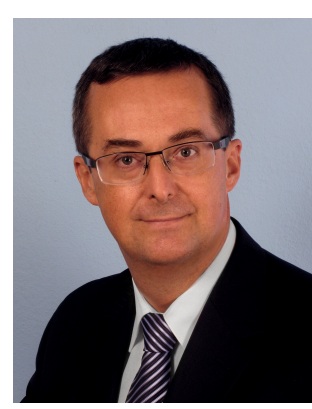

at Faculty of Mathematics and Information Science, Warsaw University of Technology, Head of Division of Artificial Intelligence and Computational Methods and Head of Doctoral Programme in Computer Science. In 2011 he was awarded the title of Professor Titular.

He is the author of 3 books and $100+$ research papers, an Associate Editor of several journals including IEEE Transactions on Computational Intelligence and AI in Games. He is a recipient of the Senior Fulbright Advanced Research Award.

His research interests include application of CI to games, dynamic optimization, human-machine cooperation, financial modeling, and development of general-purpose human-like learning and problem-solving methods which involve intuition, creativity and multitasking.

Adam Żychowski received his B.Sc. and M.Sc. de-

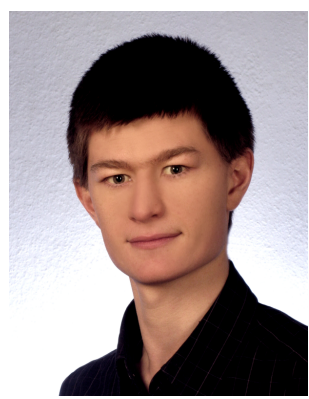
grees in Computer Science from the Faculty of Mathematics and Information Science, Warsaw University of Technology, Warsaw, Poland in 2014 and 2015, respectively.

His research interests include Computational Intelligence methods and their application to solving complex, real-life problems. 